\title{
QUALIDADE DA MADEIRA DE Cryptomeria japonica D. Don
}

\author{
Mayara Elita Carneiro ${ }^{1}$, Eduardo Bittencourt ${ }^{2}$, Graciela Ines Bolzon de Muniz ${ }^{3}$ \\ ${ }^{1} E^{2}{ }^{a}$. Industrial Madeireira, Mestranda em Eng. Florestal, UFPR, Curitiba, PR, Brasil - mayaraelita@ hotmail.com \\ ${ }^{2}$ Eng. Florestal, M.Sc., Doutorando em Recursos Naturais, University of Northern British Columbia - bittenc@unbc.ca \\ ${ }^{3}$ Eng $^{\text {a }}$. Florestal, Dr ${ }^{\text {a }}$, Depto. de Engenharia e Tecnologia Florestal, UFPR, Curitiba, PR, Brasil - gbmunize@ufpr.br
}

Recebido para publicação: 01/07/2008 - Aceito para publicação: 10/11/2008

\begin{abstract}
Resumo
A madeira de Cryptomeria japonica, apesar de não muito difundida no Brasil, é altamente valorizada em alguns países europeus, Japão e Estados Unidos. Frente a essa abordagem, o presente trabalho objetivou a caracterização da madeira de $C$. japonica plantada no Planalto Catarinense, relativamente a aspectos anatômicos, físicos e mecânicos. Os resultados obtidos mostraram valores para as propriedades estudadas em geral menores que as observadas em madeiras do gênero Pinus. Porém, quando destinada a certos usos específicos, essa madeira pode apresentar-se como uma adequada alternativa de uso.

Palavras-chave: Cryptomeria japonica; propriedades anatômicas, físicas e mecânicas.
\end{abstract}

\begin{abstract}
Wood quality of Cryptomeria japonica. Cryptomeria japonica wood, despite of not widely spread in Brazil, has a high value in some European countries, Japan and the United States. Thus, this paper had as objective the characterization of $C$. japonica wood planted in the Plateau of Santa Catarina, studying anatomic, physic and mechanical aspects. The results showed slightly smaller values for the studied properties than those observed in Pinus spp wood. However, when destined to specific uses, this wood can be an adequate alternative of utilization.

Keywords: Cryptomeria japonica; anatomical, physical and mechanical properties.
\end{abstract}

\section{INTRODUÇÃO}

A espécie Cryptomeria japonica D. Don, pertence à ordem Coniferae e à família Taxodiaceae. Originária da região temperada do Japão, onde é conhecida como "sugi", é uma das coníferas mais importantes da história japonesa, e suas plantações remotam a várias centenas de anos (CARPANEZZI; CARVALHO, 1988). No Japão, a principal justificativa para o uso da madeira produzida por essa espécie deve-se aos seus excelentes atributos (fuste reto, crescimento rápido, facilidade de processamento e cor agradável). Essa espécie é usada frequentemente em plantios comerciais no Japão, ocupando cerca de 45\% das plantações artificiais de um total de 10 milhões de hectares (OHBA, 1993). Nesse país, sua madeira vem sendo extensivamente empregada para estruturas moldadas (FUJISAWA et al., 1992).

No Brasil, a árvore é utilizada para fins ornamentais, paisagísticos e como cerca viva. Em sua fase juvenil, é comercializada como árvore de Natal. Sua característica de planta medicinal também é apontada na literatura, assim como o seu cultivo para extração de óleos essenciais para uso na indústria farmacêutica (CAPALDI, 2002).

Comercialmente, a $C$. japonica foi introduzida no Brasil pela Companhia Melhoramento e tem sido plantada há décadas, principalmente nos municípios de Caieira, no estado de São Paulo (750 a 1000 metros de altitude), e Camanducaia (1.500 metros de altitude), na Serra da Mantiqueira, no sul do estado de Minas Gerais (CARPANEZZI; CARVALHO, 1988). Para Carvalho (2001), a C. japonica é umas das espécies arbóreas alternativas para plantios florestais no Brasil, apresentando dados de incremento médio anual (IMA) em volume sólido com casca entre 14 e $45 \mathrm{~m}^{3} \cdot \mathrm{ha}^{-1}$.ano ${ }^{-1}$. Na região de coleta do material testado (Sul do Brasil), as árvores apresentam crescimento similar ou superior ao crescimento constatado nos plantios comerciais de Pinus taeda, dependendo do sítio considerado. 
A madeira de $C$. japonica é leve, com massa específica aparente entre 0,25 e $0,35 \mathrm{~g} \cdot \mathrm{cm}^{-3}$ e não apresenta grandes problemas de contração, sendo considerada uma madeira de alta durabilidade e fácil de ser trabalhada (GURGEL FILHO, 1964; CARVALHO, 2001). Além disso, é uma madeira estável, qualidade importante para processamento mecânico e para aplicações mais nobres, como movelaria (PEREIRA et al., 2003). Essa madeira pode ser utilizada para serrados em geral, assim como para celulose e papel, chapas, construções de casas, móveis, barcos e navios, caixotaria e miolo de painéis compensados, entre outros usos (SANTOS et al., 2000; GÉRARD et al., 2003). Quanto ao aspecto visual, a madeira apresenta alburno amarelo-claro e cerne marrom-escuro, com textura fina e grã reta (PEREIRA et al., 2003; GÉRARD et al., 2003).

De maneira geral, $C$. japonica é uma espécie que apresenta grande potencial para plantios nas regiões altas e frias do Brasil, destacando-se pelo seu rápido crescimento, boa adaptação ao clima e solo do Sul do país, boa resposta às tecnologias silviculturais, qualidade e diversidade de utilização de sua matériaprima para indústrias madeireiras (SANTOS et al., 2000). Mesmo com o descrito potencial, literaturas científicas que abordem os aspectos tecnológicos da madeira de $C$. japonica são escassas. Dessa forma, o presente trabalho avaliou algumas propriedades anatômicas, físicas e mecânicas da madeira de Cryptomeria japonica plantadas no Sul do Brasil, como forma de potencializar o uso da espécie.

\section{MATERIAL E MÉTODOS}

Foram utilizadas 15 árvores de C. japonica, de plantios comerciais com 14 anos de idade, localizados na fazenda Guamirim Gateados, da Empresa Florestal Gateados Ltda., no município de Campo Belo do Sul, estado de Santa Catarina. As árvores foram selecionadas ao acaso, primando pela representatividade da população. As árvores abatidas foram seccionadas em toras de 2,5 metros de comprimento e seguindo a norma D 5536-94 da ASTM (1995a). De cada tora foi retirado um pranchão central com 8,0 centímetros de espessura. Para esse estudo, apenas a primeira tora de cada árvore foi utilizada para a obtenção de amostras.

O material coletado foi transportado para o Laboratório de Propriedades Físicas e Mecânicas da Madeira da Universidade do Planalto Catarinense (UNIPLAC), onde os pranchões foram gradeados para uma secagem lenta e gradual. Depois de atingirem a umidade de cerca de $15 \%$, os pranchões foram convertidos em corpos de prova para o ensaio das propriedades anatômicas, físicas e mecânicas da madeira.

Tanto para descrição anatômica quanto para as mensurações dos elementos celulares individuais, seguiram-se recomendações feitas por Muniz; Coradin (1991) e Iawa (2004). Para análise anatômica, foram retirados no sentido radial da faixa central do pranchão cinco corpos de prova (medula, 1/4, 2/4, $3 / 4$ e 4/4 do raio) por tora. A descrição da estrutura microscópica do lenho foi realizada a partir da análise dos cortes histológicos da madeira em lâminas permanentes e da dissociação do lenho.

Para avaliação da massa específica e da instabilidade dimensional, seguiu-se a norma ASTM D2395 (1997b). Inicialmente os corpos de prova foram climatizados em um ambiente normalizado $\left(20^{\circ} \mathrm{C}\right.$ de temperatura e $65 \%$ de umidade relativa), até atingirem a umidade de equilíbrio de $12 \%$, quando foram pesados e mensurados. Após esse passo, fez-se a saturação dos corpos de prova em água, utilizando-se o método de imersão em dessecadores com sistema de vácuo até a estabilização do peso dos corpos de prova, quando novamente foram pesados e mensurados. Em seguida, os corpos de prova foram colocados para secar em estufa a $103 \pm 2{ }^{\circ} \mathrm{C}$. Após a estabilização dos pesos, eles foram mensurados de forma equivalente. Assim, a massa específica básica foi determinada pela razão entre a massa seca e o volume saturado determinado em g. $\mathrm{cm}^{-3}$. Já a massa específica aparente a $12 \%$ foi determinada através da razão entre a massa e o volume, ambos ao teor de umidade de $12 \%$. A instabilidade dimensional da madeira, expressa nas contrações longitudinal, radial, tangencial e volumétrica, mede a diferença na dimensão dos corpos de prova quando estão completamente saturados para completamente secos - os referidos valores foram obtidos em termos percentuais.

A caracterização mecânica foi realizada através da avaliação da dureza paralela à fibra, dureza paralela ao raio, dureza perpendicular ao raio, cisalhamento, compressão paralela às fibras e flexão estática. Os procedimentos de confecção, condicionamento e ensaio dos corpos de prova seguiram a recomendação D 143-94 da norma ASTM (1995c). Os corpos de prova foram climatizados em um ambiente normalizado $\left(20^{\circ} \mathrm{C}\right.$ de temperatura e $65 \%$ de umidade relativa), até atingirem a umidade de 
equilíbrio de aproximadamente $12 \%$. Nessa condição foram realizados os testes mencionados, em uma máquina universal de ensaios EMIC, servo-controlada e dotada de acessórios específicos para cada teste, pertencente ao Laboratório de Propriedades Físicas e Mecânicas da Madeira da UNIPLAC.

Como procedimento básico de análise dos resultados, foram calculados os valores médios, o desvio padrão e o coeficiente de variação para cada propriedade testada.

\section{RESULTADOS E DISCUSSÃO}

\section{Propriedades anatômicas da madeira}

A madeira de $C$. japonica macroscopicamente apresenta cerne e alburno distintos pela cor. O alburno é amarelo-claro e o cerne marrom-escuro, de brilho moderado. Tem textura fina, grã direita e é macia ao corte.

A figura 1 apresenta as fotomicrografias dos cortes histológicos das secções transversal, radial e tangencial do tecido de C. japonica. Microscopicamente, essa espécie apresenta os anéis de crescimento distintos, com a transição do lenho inicial para o tardio gradual a abrupta. As pontoações são areoladas, dispostas em fileiras unisseriadas, com pontoações de campo de cruzamento do tipo taxodioide. Os raios apresentam-se de forma predominante unisseriados baixos - a maioria entre 3 a 8 células de altura. $\mathrm{O}$ parênquima axial é disposto na forma de agregados tangenciais, com parede transversal e espessamento irregular.

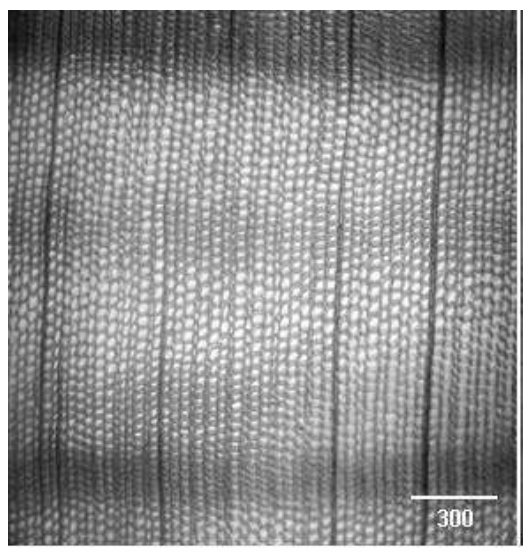

A

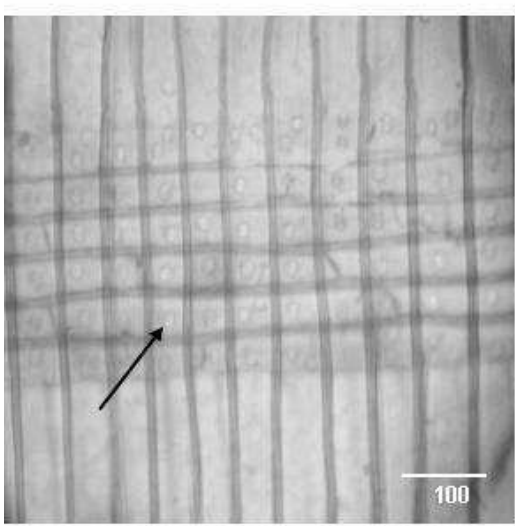

$\mathrm{C}$

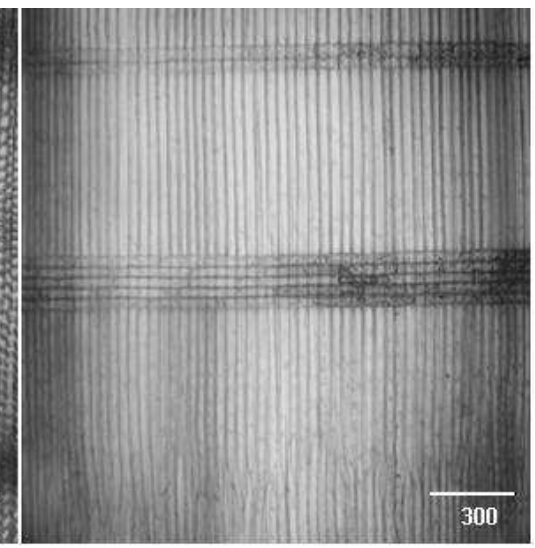

B

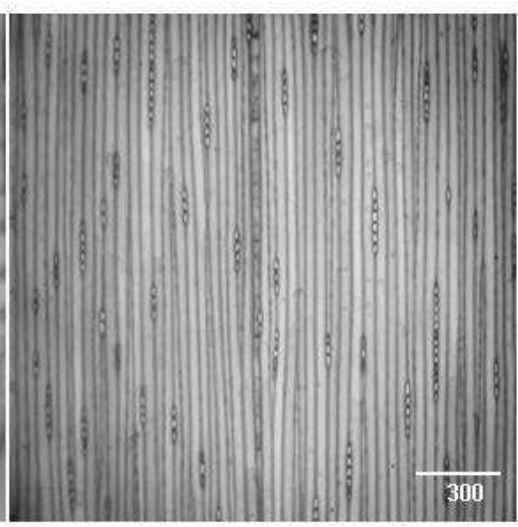

D

Figura 1. Fotomicrografias de cortes histológicos de Cryptomeria japonica. A. secção transversal. B. Secção radial. C. Secção radial, detalhe campo de cruzamento com pontoações tipo taxodioide. D. Secção tangencial.

Figure 1. Photomicrography of histological cut of Cryptomeria japonica. A. Transversal section. B. Radial section. C. Radial section, detail cross-field pit type Taxodioid. D. Tangential section. 
Os valores médios das características morfológicas dos traqueoides axiais são apresentados na tabela 1. Os valores médios para comprimento dos traqueoides determinados neste estudo são pouco inferiores aos observados em outros estudos com a mesma espécie em indivíduos mais velhos. Pereira $e t$ al., (2003) encontraram valores médios para comprimento dos traqueoides de 2,21 $\mathrm{mm}$ para oito árvores de 22 anos de idade de Colombo (PR). Colodette (1982) encontrou valores médios para comprimento dos traqueoides de $2,24 \mathrm{~mm}$.

Tabela 1. Características morfológicas médias dos traqueoides axiais da madeira de Cryptomeria japonica.

Table 1. Average morphologic characteristics of the axial tracheid of Cryptomeria japonica wood.

\begin{tabular}{lcccc}
\hline & $\begin{array}{c}\text { Comprimento } \\
(\mathbf{m m})\end{array}$ & $\begin{array}{c}\text { Largura } \\
(\boldsymbol{\mu} \mathbf{m})\end{array}$ & $\begin{array}{c}\text { Diâmetro } \\
\text { lume }(\boldsymbol{\mu m})\end{array}$ & $\begin{array}{c}\text { Espessura da } \\
\text { parede }(\boldsymbol{\mu m})\end{array}$ \\
\hline Média & 2,15 & 29,4 & 18,6 & 5,3 \\
Desvio padrão & 0,08 & 2,8 & 3,1 & 0,4 \\
Coef. variação $(\%)$ & 3,95 & 9,6 & 16,6 & 6,7 \\
\hline
\end{tabular}

Nas literaturas consultadas, pode-se observar que os comprimentos médios dos traqueoides da $C$. japonica, em idades próximas, são menores que os de Pinus taeda e Pinus elliottii. Nisgoski (2005) obteve média para comprimento dos traqueoides de $P$. taeda com idade de 11 anos de $3,20 \mathrm{~mm}$, e Barrichelo (1984), com P. elliottii com idade compreendida entre 10 e 12 anos, obteve um valor médio para comprimento dos traqueoides de $3,86 \mathrm{~mm}$.

Os valores obtidos para largura são inferiores aos verificados por outros autores trabalhando com a mesma espécie. Pereira et al. (2003) obtiveram média para a largura de 30,1 $\mu \mathrm{m}$, enquanto Colodette (1982) encontrou média para a largura de $31,0 \mathrm{~mm}$. Em ambos os estudos obtiveram-se médias um pouco maiores que as observadas nesta pesquisa. Comparando os dados da variação da largura média dos traqueoides de C. japonica com espécie do gênero Pinus, Foelkel (1976) observou valores de 43,81 $\mu \mathrm{m}$ para $P$. taeda de 11 anos. Barrichelo (1984), com P. elliottii com idade compreendida entre 10 e 12 anos, obteve média de $45,8 \mu \mathrm{m}$.

Em relação ao diâmetro médio do lume, os dados obtidos nesta pesquisa são também inferiores aos relatados na literatura para a mesma espécie. Pereira et al. (2003) obtiveram média de 19,2 $\mu \mathrm{m}$, e Colodette (1982) encontrou média de 21,8 $\mu \mathrm{m}$. Ao correlacionar os dados desta pesquisa com o diâmetro de lume para o gênero Pinus, novamente foi evidenciado que para idades próximas as dimensões morfológicas são maiores, como apontado por Klock (2000), que verificou uma média de 32,9 $\mu \mathrm{m}$ no lenho inicial e 22,3 $\mu \mathrm{m}$ no lenho tardio para $P$. taeda com 11 anos de idade. Foelkel (1976) observou valores de 34,28 $\mu \mathrm{m}$ para $P$. taeda de 11 anos. Barrichelo (1984), trabalhando com P. elliottii com idade compreendida entre 10 e 12 anos, obteve média de $28,2 \mu \mathrm{m}$.

Para espessura da parede, os resultados são compatíveis aos relatados na literatura para a mesma espécie. Pereira et al., (2003) obteve média de 5,5 $\mu \mathrm{m}$. Colodette (1982) encontrou média de 4,8 $\mu \mathrm{m}$. Ao comparar as características morfológicas dos traqueoides da $C$. japonica com espécies do gênero Pinus, verificam-se valores mais compatíveis aos de $P$. taeda, como apontado por diversos autores. Klock (2000) verificou uma média de 4,7 $\mu \mathrm{m}$ no lenho inicial e $8,1 \mu \mathrm{m}$ no lenho tardio para $P$. taeda com 11 anos de idade. Foelkel (1976) observou valores de 4,96 $\mu \mathrm{m}$ para $P$. taeda de 11 anos, e Barrichelo (1984), trabalhando com $P$. elliotti com idade compreendida entre 10 e 12 anos, obteve média de $8,8 \mu \mathrm{m}$.

Para todas as mensurações anatômicas realizadas, observou-se um aumento na variabilidade radial, fato típico para madeira juvenil de espécies do gênero Pinus. Essa é uma tendência descrita por diversos autores, como Zobel et al. (1959), Duffield (1964), Burley (1973) e Klock et al. (2002), entre outros.

\section{Propriedades físicas da madeira}

As propriedades físicas da madeira avaliadas nesse estudo - massa específica básica, massa específica aparente, índices de contrações e coeficiente de anisotropia - são apresentadas na tabela 2 em valores médios. 
Tabela 2. Valores médios de massa específica (básica e aparente a 12\%) e índices de contrações da madeira de Cryptomeria japonica.

Table 2. Mean values for specific gravity (ovendry and 12\%) and shrinkage of Cryptomeria japonica wood.

\begin{tabular}{lcccccc}
\hline & \multicolumn{2}{c}{ Massa específica (g.cm) } & \multicolumn{3}{c}{ Contrações (\%) } & Coeficiente de \\
\cline { 2 - 6 } & Básica & Aparente & Tangencial & Radial & Volumétrica & Anisotropia \\
\hline Média & 0,255 & 0,307 & 6,59 & 2,96 & 9,96 & 2,54 \\
Desvio Padrão & 0,024 & 0,026 & 1,68 & 1,54 & 2,96 & 0,77 \\
Coef. Variação (\%) & 9,424 & 8,531 & 25,55 & 52,05 & 29,69 & 30,13 \\
\hline
\end{tabular}

Para os valores de massa específica básica da madeira de $C$. japônica, destaca-se a uniformidade de distribuição dos valores obtidos. Os valores médios encontrados neste estudo foram inferiores aos resultados encontrados por outros pesquisadores que trabalharam com a mesma espécie. Pereira et al. (2003) encontraram valores médios para massa específica básica de $0,360 \mathrm{~g} . \mathrm{cm}^{-3} \mathrm{em}$ árvores de 22 anos de idade.

Comparando-se as características da madeira de C. japonica cultivada no Japão e no Brasil, pode-se observar que os valores de massa específica da madeira apresentam semelhanças. Ishiguri et al. (2005), trabalhando com diferentes espaçamentos e tratos silviculturais em árvores com 29 anos de idade, obtiveram valores de 0,360 g.cm ${ }^{-3}$ para massa específica básica. De maneira geral, os valores da $C$. japonica foram inferiores aos observados em Pinus. Para P. taeda, Klock (2000) encontrou valores médios de massa específica básica de $0,420 \mathrm{~g} . \mathrm{cm}^{-3}$ para árvores de 11 anos de idade, Bittencourt (2004) obteve média de 0,366 g.cm ${ }^{-3}$ para árvores de 14 anos e Nisgoski (2005) obteve média de 0,354 g.cm ${ }^{-3}$ para árvores de 15 anos.

Os índices de contração volumétrica relatados por Gérard et al. (2003) e Pereira et al. (2003) foram de 9,24\% e 10,1\%, valores próximos aos encontrados neste estudo. Comparando os valores de $C$. japonica com espécies do gênero Pinus, Muñiz (1993) encontrou valores de contração volumétrica total de $10,7 \%$ em P. elliottii e $10,9 \%$ em P. taeda.

O valor do coeficiente de anisotropia para este estudo foi de 2,54, estando próximo aos valores relatados por Gérard et al. (2003) e Pereira et al. (2003). Porém, se comparado com valores de P. elliottii e $P$. taeda (1,75 e 1,76, respectivamente), obtidos por Muñiz (1993), tem-se maiores valores na $C$. japonica. Isso indica uma maior instabilidade dimensional para essa madeira quando submetida à secagem.

\section{Propriedades mecânicas da madeira}

Os valores obtidos para propriedades mecânicas testadas encontram-se na tabela 3.

Tabela 3. Resultados médios das propriedades mecânicas da madeira de Cryptomeria japonica.

Table 3. Mean results of mechanical properties of Cryptomeria japonica wood.

\begin{tabular}{|c|c|c|c|c|c|c|c|}
\hline & \multirow{2}{*}{$\begin{array}{c}\text { Cisalhamento } \\
\left(\text { kgf.cm }^{-2}\right)\end{array}$} & \multicolumn{2}{|c|}{$\begin{array}{l}\text { Flexão Estática } \\
\left(\text { kgf.cm }^{-2}\right)\end{array}$} & \multirow{2}{*}{$\begin{array}{c}\text { Compressão } \\
\text { MOE } \\
\left(\text { kgf.cm }^{-2}\right)\end{array}$} & \multicolumn{3}{|c|}{ Dureza Janka (kgf.cm²) } \\
\hline & & MOE & MOR & & $\mathbf{A}$ & $T$ & $\mathbf{R}$ \\
\hline Média & 61,5 & 56.484 & 387,4 & 2.302 & 234,8 & 136,3 & 126,7 \\
\hline Desvio Padrão & 7,8 & 15.571 & 81,4 & 292 & 36,8 & 27,4 & 34,3 \\
\hline Coef. Variação (\%) & 12,7 & 27,6 & 21,0 & 12,7 & 15,7 & 20,1 & 27,0 \\
\hline
\end{tabular}

A: face axial ou transversal; $\mathrm{T}$ : face tangencial; R: face radial.

Não foram encontrados dados na literatura para valores da madeira de C. japonica para resistência ao cisalhamento. Porém os valores obtidos foram inferiores aos observados em outras coníferas, como em Santini et al. (2000), cujos valores médios para $P$. elliottii foram $85 \mathrm{kgf.cm}^{-2}$ e 87 kgf.cm ${ }^{-2}$ para $P$. taeda.

Os valores encontrados no módulo de elasticidade (MOE) para flexão estática na $C$. japonica nesta pesquisa foram pouco inferiores aos encontrados por Gérard et al. (2003), que relatam o valor de $59.449 \mathrm{kgf.cm}^{2}$. Santini et al. (2000) relatam valores médios para o módulo de ruptura (MOR) de $555 \mathrm{kgf.cm}{ }^{-2}$ para $P$. elliottii e $556 \mathrm{kgf.cm}^{-2}$ para $P$. taeda. Para o Módulo de Elasticidade (MOE), os 
valores foram de $73.266 \mathrm{kgf.cm}{ }^{-2}$ para $P$. elliottii e $87.606 \mathrm{kgf.cm}^{-2}$ para $P$. taeda, ambas as espécies com 19 anos de idade.

Para a resistência da madeira à compressão paralela às fibras, o valor médio encontrado para o módulo de elasticidade foi inferior ao observado por Santini et al. (2000), com valores médios de 2.595 kgf.cm ${ }^{-2}$ para $P$. elliottii e $2.960 \mathrm{kgf.cm}{ }^{-2}$ para $P$. taeda, em árvores com 19 anos.

Os valores observados neste estudo para dureza nas faces axial, tangencial e radial foram inferiores aos observados em outras coníferas. Santini et al. (2000) relatam valores médios para P. elliottii de 366 kgf.cm ${ }^{-2}, 293 \mathrm{kgf.cm}{ }^{-2}$ e $224 \mathrm{kgf.cm}^{-2}$, respectivamente, nas faces axial, tangencial e radial, enquanto que para $P$. taeda os mesmos valores foram $315 \mathrm{kgf.cm}^{-2}, 302 \mathrm{kgf.cm}^{-2}$ e $222 \mathrm{kgf. \textrm {cm } ^ { - 2 }}$.

\section{CONCLUSÕES}

No geral, os valores médios observados no presente estudo foram inferiores àqueles citados na literatura, o que pode ser atribuído primeiramente à característica juvenil da madeira. Árvores originárias de povoamentos maduros, devido à sua grande proporção de madeira adulta, possuem maior resistência mecânica que árvores de povoamentos jovens. A presença de células relativamente mais longas e de paredes mais espessas, bem como a maior proporção de lenho tardio, confere à madeira adulta maior massa específica e, consequentemente, propriedades de resistência mais elevadas. Nesse caso, certas diferenças em propriedades anatômicas, físicas e mecânicas são previamente esperadas.

A caracterização da madeira de C. japonica permite obter prováveis comportamentos tecnológicos em processos industriais em que se tem a madeira como matéria-prima. Em termos de suas características elementais, a madeira de $C$. japonica plantada na região do Planalto Catarinense apresenta traqueoides com comprimento e diâmetro do lume pouco menores que os observados em outros estudos com a mesma espécie. Isso se deve ao fato de que a idade estudada (14 anos) era menor que as estudadas por outros autores (22 anos). Conforme descrito por Walker (1993) e Desch; Dinwoodie (1996), essa variabilidade nas dimensões dos traqueoides é uma característica padrão para coníferas. Vislumbrando-se o uso da madeira no processo de polpação e fabricação de papel, tendo as características anatômicas como base, é esperado um papel com altas propriedades de alongamento, resistência ao estouro, resistência à passagem de ar e absorção de energia de tensão. Por outro lado, a resistência ao rasgo seria diminuída - as extrapolações são baseadas na polpa produzida a partir de Pinus taeda de semelhante idade. As características previstas vão ao encontro do obtido por Colodette (1992), o qual, apesar do rendimento de polpação menor que o de $P$. elliottii, destaca que o material apresenta características compatíveis com o uso em processo kraft.

Os resultados das propriedades físicas obtidas neste estudo indicaram uma menor massa específica em relação a outros estudos com a mesma espécie e também quando comparados com a madeira do gênero Pinus. A menor massa específica observada é o resultado da idade e de um crescimento mais acelerado da espécie na condição estudada. De maneira geral, a madeira apresentou-se menos densa e com maior anisotropia de contração que as madeiras do gênero Pinus com idade próxima. Isso indica um provável comportamento de baixo tempo total de secagem e maior tendência de ocorrência de defeitos devido à secagem que os observados em geral em madeiras de Pinus.

As propriedades mecânicas estudadas demonstraram que a espécie apresentou valores de resistência menores que os encontrados na literatura para $C$. japonica e para o gênero Pinus. Os valores encontrados indicam uma madeira não adequada para fins estruturais ou mesmo usos que requeiram dureza superficial, como em pisos de madeira. Porém essas características induzem seu uso como material isolante, miolo de painéis, portas e outros usos em que certa resistência mecânica seja requerida, mas a característica de baixa massa específica seja mais importante.

De forma genérica, a espécie $C$. japonica vem demonstrando grande potencial adaptativo às condições de plantio do Planalto Catarinense, destacado especialmente pelo seu crescimento. Associado a esse fator, as características da madeira juvenil apresentaram-se promissoras tanto em aspectos anatômicos e físicos quanto mecânicos. Se a madeira for destinada para usos que requeiram pequenos traqueoides (como papéis de alto acabamento), baixa massa específica (como material com baixa condutividade térmica, elétrica ou acústica), ou ainda baixa resistência mecânica (painéis isolantes ou miolos de produtos recompostos), a $C$. japonica oferecerá uma matéria-prima com boa adaptabilidade. Para cada uso desejado, testes específicos devem ser feitos para o ajuste de condições operacionais ou eventuais incompatibilidades, como quantidade de extrativos presentes ou resistência à degradação. 


\section{REFERÊNCIAS}

AMERICAN SOCIETY FOR TESTING AND MATERIALS (ASTM). D 5535-94: Practice for sampling forest trees for determination of clear wood properties. Philadelphia, 1995a.

1995b.

D 2395-93: Test methods for specific gravity of wood and wood-base materials. Philadelphia,

D 143-94: Standard methods of testing small clear specimens of timber. Philadelphia, 1995c.

BARRICHELO, L. E. G. Caracterização de madeiras de Pinus spp. Boletim Informativo IPEF, Piracicaba, v. 3, n. 14, p. 100-112, 1984.

BITTENCOURT, E. Parâmetros de otimização no processo de fabricação de celulose e papel. $61 \mathrm{f}$. Dissertação (Mestrado em Engenharia Florestal) - setor de Ciências Agrárias, Universidade Federal do Paraná, Curitiba. 2004.

BURLEY, J.; NIKLES, D. G. Summary report on variation in density and tracheid length on ten trees of Pinus caribaea var. hondurensis Barr. and Golf. from Jamaica, 1971. In: BURLEY, J.; NIKLES, D.G. Selection and breeding to improve some tropical conifers. Oxford, Commonwealth Forestry Institute, 1973. p. 72-85.

CAPALDI, F. R. Avaliação de diferentes fontes de nitrogênio em explantes de Cryptomeria japonica D. DON. "ELEGANS" cultivadas in vitro: análises bioquímicas e relações entre reguladores vegetais. Dissertação (Mestrado) - Escola Superior de Agricultura "Luiz de Queiroz"; Universidade de São Paulo, Piracicaba. 2002.

CARPANEZZI, A.; CARVALHO, P. E. R. Zoneamento ecológico para plantios florestais do Estado de Santa Catarina. Curitiba: EMBRAPA-CNPF, 1988. 103 p. (Documento, n. 21).

CARVALHO, P. E. R. Novas alternativas para reflorestamento. Revista da Madeira, Curitiba, ano 11, $\mathrm{n}$. 60, p. 66 a 67, 2001.

COLODETTE, J. L. Estudo das características da madeira e polpa kraft da Cryptomeria japonica D. Don., In: CONGRESSO ANUAL DA ABCP, 1, 1982, São Paulo. Anais... São Paulo, 1982. p. 139-155.

DESH, H. E.; DINWOODIE, J. M. Timber: structure, properties, conversion and use. 7. ed. New York: Food products press, 1996. p. 306.

DUFFIELD, J. W. Tracheid length variation patterns in douglas-fir and selection of extreme variants. TAPPI, Atlanta, v. 47, n. 2, p. 122-8, 1964.

FOELKEL, C. E. B.; BARRICHELO, L. E. G., GARCIA, W.; BRITO, J. O. Celulose Kraft de madeiras juvenil e adulta de Pinus elliottii. IPEF. Piracicaba. n. 12, p. 127-142. 1976.

FUJISAWA, Y.; OHTA, S.; NISHIMURA, K.; TAJIMA, M. Wood characteristics and genetic variation in sugi (Cryptomeria japonica), clonal differences and correlations between plustree clones. Mokuzai Gakkaishi, Tokyo, v. 38, p. 638-644, 1992.

GÉRARD, J.; BEAUCHÊNE, J.; FOUQUET, D.; GUIBAL, D.; LANGBOUR, P.; THEVENON, M. F.; THIBAUT, A.;VERNAY, M. TROPIX 5.0: Caractéristiques technologiques de 215 essences tropicales. Montpellier: CIRAD-Forêt, 2003. Disponível <http://www.cirad.fr/activities/bois/ew/syst/asia/sugi.pdf>. Acesso em: 13/10/2006.

GURGEL FILHO, O. A. O comportamento florestal das coníferas exóticas. Silvicultura em São Paulo, São Paulo, v. 3, n. 3, p. 129-188, 1964.

INTERNATIONAL ASSOCIATION OF WOOD ANATOMISTS (IAWA). IAWA List of Microscopic features for softwood identification. IAWA Journal, Leiden, v. 25, p. 1-70, feb. 2004.

ISHIGURI, F.; KASSAI, S.; YOKOTA, K.; YOSHIZAWA, N. Wood Quality of Sugi (Cryptomeria japonica) grown at four initial spacings. IAWA Journal, Leiden, v. 26, n. 3, p. 375 - 385, 2005. 
KLOCK, U. Qualidade da madeira juvenil de Pinus maximinoi H. E. Moore. 291 f. Tese (Doutorado em Engenharia Florestal) - Setor de Ciências Agrárias, Universidade Federal do Paraná, Curitiba, 2000.

KLOCK, U.; MUÑIZ, G. I. B.; BITTENCOURT, E.; ANDRADE, A. S.; Densidade básica da madeira ao longo do fuste e entre classes diamétricas de Pinus taeda. VIII Encontro Brasileiro em Madeiras e Estruturas de Madeira. Uberlândia. v. 1, p. 10- 20. 2002. Disponível em: <http://www.celuloseonline.com.br/imagembank/Docs/DocBank/dc/dc391.pdf>. Acesso em: 02/03/2007.

MUÑIZ, G. I. B. Caracterização e desenvolvimento de modelos para estimar as propriedades e o comportamento na secagem da madeira de Pinus elliottii Engelm. e Pinus taeda L. 235 f. Tese (Doutorado em Ciências Florestais) - Universidade Federal do Paraná, Setor de Ciências Agrárias, Curitiba, 1993.

MUNIIZ, G. I. B.; CORADIN, V. R. Norma de procedimentos em estudos de anatomia da madeira: II Gimnospermae. Brasília, DF: ABNT, 1991. (Série técnica. Laboratório de Produtos Florestais). Comissão de estudos CE:11.01.07:002.

NISGOSKI, S. Espectroscopia no infravermelho próximo no estudo de características da madeira e papel de Pinus taeda L. 160 f. Tese (Doutorado em Engenharia Florestal) - Setor de Ciências Agrárias, Universidade Federal do Paraná, Curitiba, 2005.

OHBA, K. Clonal Forestry with sugi (Cryptomeria japonica). In: AHUJA, M. R.; LIBBY, W. J. (Eds). Clonal forestry II: conservation and application. Berlin: Springer Verlag, 1993. p. 66-90.

PEREIRA, J. C. D.; HIGA, R. C. V.; SHIMIZU, J. Y. Propriedades da madeira do cedrinho japonês. Colombo: Embrapa Florestas, 2003. 4 p. (Comunicado técnico, n. 88).

SANTOS, C. B.; LONGHI, S. J.; HOPPE, J. M.; MOSCOVICH, S. A. Efeito do volume de tubetes e tipos de substratos na qualidade de mudas de Cryptomeria japonica (L.F.) D. Don. Ciência Florestal, Santa Maria, v. 10, n. 2, p. 1-15, 2000.

SANTINI, H. S.; HASELEIN, C. R.; GATTO, D. A. Análise Comparativa das Propriedades Físicas e Mecânicas da Madeira de Três Coníferas de Florestas Plantadas. Ciência Florestal, Santa Maria, v. 10, n. 1, p. 85-93, 2000.

WALKER, J. C. F. Primary wood processing: principle and practice. Londres: Chapman \& Hall, 1993. p. 595.

ZOBEL, B. J.; WEBB, C.; HENSON, F. Core or juvenile wood of loblolly pine trees. TAPPI, Atlanta, v. 42 , n. 5, p. 345-56, 1959. 